\title{
Preclinical evaluation of cisplatin-incorporated bio-nanocapsules as chemo-radiotherapy for human hepatocellular carcinoma
}

\author{
SEOL HWA SHIN ${ }^{1,2}$, SEOK SOON PARK ${ }^{1,2}$, KYOUNG JIN LEE ${ }^{1,2}$, EUN JIN JU ${ }^{1,2}$, JIN PARK ${ }^{1,2}$, \\ EUN JEONG KO ${ }^{1,2}$, JOOHEE JUNG ${ }^{3,4}$, SHUN'ICH KURODA ${ }^{5}$, SEUNG-MO HONG ${ }^{6}$, JUNG JIN HWANG ${ }^{1,2,7,10}$, \\ JUNG SHIN LEE ${ }^{1,8}$, SI YEOL SONG ${ }^{1,9,10}$, SEONG-YUN JEONG ${ }^{1,2,7,10}$ and EUN KYUNG CHOI ${ }^{1,9,10}$ \\ ${ }^{1}$ Institute for Innovative Cancer Research, ${ }^{2}$ ASAN Institute for Life Sciences, ASAN Medical Center, University of \\ Ulsan College of Medicine, Seoul 05505; ${ }^{3}$ College of Pharmacy, and ${ }^{4}$ Innovative Drug Center, Duksung Women's \\ University, Seoul 01369, Republic of Korea; ${ }^{5}$ Department of Bioengineering Science and Reaction, The Institute \\ of Scientific and Industrial Research (ISIR-Sanken) Osaka University, Osaka 567-0046, Japan; Departments of \\ ${ }^{6}$ Pathology, ${ }^{7}$ Convergence Medicine, ${ }^{8}$ Internal Medicine, ${ }^{9}$ Radiation Oncology, ${ }^{10}$ Center for Advancing Cancer \\ Therapeutics, ASAN Medical Center, University of Ulsan College of Medicine, Seoul 05505, Republic of Korea
}

Received April 5, 2017; Accepted July 24, 2017

DOI: $10.3892 / o r .2017 .5910$

\begin{abstract}
The incidence of hepatocellular carcinoma (HCC) has continued to increase worldwide, and advanced HCC is difficult to treat using the currently available therapeutics. Chemoradiotherapy with cisplatin (cis-diamminedichloroplatinum, CDDP) is expected to confer a curative benefit on HCC patients; however, its application is limited due to side-effects such as acute nephrotoxicity as well as the conventionally limited application of chemoradiotherapy for HCC. For the practical application of this drug in the clinical setting, we formulated a novel drug carrier-comprising bio-nanocapsule (BNC) and liposomal CDDP (BNC-LP-CDDP) that recognizes the human liver and releases CDDP. BNC-LP-CDDP showed selectively high cytotoxicity for $\mathrm{HCC}$ cells, and markedly reduced the survival fractions of HCC when combined with ionizing radiation (IR) treatment in in vitro assays. In particular, the treatment of mice bearing human HCC with BNC-LP-CDDP and 3 Gy IR showed $95.68 \%$ growth inhibition, whereas IR treatment alone showed $65.6 \%$ growth inhibition. Moreover, BNC-LP-CDDP led to the withdrawal of CDDP-induced nephrotoxicity. These results indicate that BNC-LP-CDDP in combination with IR markedly enhanced the chemo-radiotherapeutic efficacy and eliminated CDDP
\end{abstract}

Correspondence to: Professor Eun Kyung Choi, Department of Radiation Oncology, ASAN Medical Center, University of Ulsan College of Medicine, Seoul 05505, Republic of Korea

E-mail: ekchoi@amc.seoul.kr

Professor Seong-Yun Jeong, ASAN Institute for Life Sciences and Department of Convergence Medicine, ASAN Medical Center, University of Ulsan College of Medicine, Seoul 05505, Republic of Korea

E-mail: syj@amc.seoul.kr

Key words: hepatocellular carcinoma, radiotherapy, bio-nanocapsule, cisplatin, liposome induced nephrotoxicity, thus, suggesting the potential for its clinical application as human HCC therapy.

\section{Introduction}

Hepatocellular carcinoma (HCC) is the fifth most common cancer and the third most common cause of cancer-related death worldwide $(1,2)$. Surgical resection, liver transplantation, percutaneous ethanol injection (PEI), and radiofrequency ablation (RFA) are considered to be the primary treatments for patients with possibly curable disease. Sorafenib is another currently available treatment option for HCC, but its efficacy is not satisfactory due to insufficient anticancer properties in a large portion of HCC patients (3).

Radiotherapy is one of the most effective tools in the clinical treatment of many types of solid tumors; however, its use for $\mathrm{HCC}$ has been limited due to the intrinsic tolerance of the liver and the development of radiation-induced liver disease $(4,5)$. As an increase in the radiation dose may lead to severe damage to normal tissues around the tumor and could potentially result in wound complications and transformation of tumor to radioresistant ones $(6,7)$, this cannot serve as an option for enhancing the response of cancers to radiation therapy. Ideally, effective radiation therapy should eliminate cancer cells in the tumor and simultaneously protect normal tissues and organs (8). Recent studies have shown that drug delivery systems, such as radiosensitizer-incorporated nanocarriers capable of targeting tumors, can enhance radiosensitivity (9-11). Murine monoclonal anti-EGFR antibody-conjugated gold nanoparticle (AuNP) containing $\beta$-lapachone, an anticancer drug that induces direct cytotoxic effects and loss of telomerase activity, appears to enhance radiosensitivity through active targeting of the tumor in vitro and in vivo (9). Polymeric nanoparticles containing taxanes, an anti-neoplastic agent, or sirolimus, an mTOR inhibitor (PNP-taxanes or PNP-sirolimus, respectively), reportedly enhanced the efficacy of chemoradiation therapy in non-small cell lung cancer $(10,11)$. 
Cis-diamminedichloroplatinum (II) (cisplatin, CDDP) is a chemotherapeutic drug used for the treatment of various solid tumors and is a well-known radiosensitizing agent (12-14). CDDP binds to DNA through crosslinking, induces DNA damage and then triggers apoptosis, if the damage is not repaired. Although CDDP is preferentially used for HCC treatment, it also evokes side-effects such as nephrotoxicity, hematopoietic injury, and deafness through a biotransformation pathway $(15,16)$. Nephrotoxicity is the most severe among these side-effects and is responsible for the dose limitation of this drug. Although the incorporation of CDDP into nanoparticles to overcome this limitation has been proposed $(17,18)$, this option has not been tested for HCC or as an active targeting drug delivery system.

We recently created a bio-nanocapsule (BNC), a hollow particle $\sim 50 \mathrm{~nm}$ in diameter, that contained the hepatitis $B$ virus surface antigen ( $\mathrm{HBsAg}$ ) L protein and displayed a human hepatocyte-recognizing molecule (pre-S1 peptide), and reported on its effectiveness for active targeting to human liver (19). BNC binds well with the surface of the liposome (LP), probably due to the spontaneous fusion of the lipid bilayer of the BNC with the LP $(20,21)$. Since LPs can adjust both the pharmacokinetics and biodistribution of the drugs by modifying the size, surface charge, and membrane lipid packing, it has become widely accepted as a well-characterized, classical carrier for drug delivery (22). A size-controlled LP can efficiently deliver a drug to the tumor site through the enhanced permeability and retention (EPR) effect and can protect the drug from metabolic processes that could clear it from the body (23). However, LPs may not be able to actively target a specific site or ensure long-term circulation in the bloodstream due to its rapid elimination through the reticuloendothelial system (RES) (24). Therefore, we used BNC and LP as a fused complex to achieve active targeting drug delivery specifically aimed at HCC based on the characteristics of BNC and LP.

In the present study, we introduced a BNC-LP complex that incorporates CDDP (BNC-LP-CDDP) for clinical application in human $\mathrm{HCC}$ treatment, and preclinically proved its effectiveness through in vitro and in vivo analysis.

\section{Materials and methods}

Preparation and characterization of BNC-LP-CDDP. BNC was prepared as previously described $(21,25)$. In brief, BNCs were purified from $S$. cerevisiae AH22R cells harboring the BNC expression plasmid, pGLDLIIP39-RcT. Protein concentrations were determined with a bicinchoninic acid (BCA) protein assay kit (Thermo Fisher Scientific, Inc., Rockford, IL, USA) using bovine serum albumin (BSA) as a control protein. To remove contaminated materials, all samples were precipitated with ice-cold acetone containing $10 \%(\mathrm{w} / \mathrm{v})$ trichloroacetic acid and $0.07 \%(\mathrm{v} / \mathrm{v}) 2$-mercaptoethanol at $-20^{\circ} \mathrm{C}$ for $1 \mathrm{~h}$. The precipitated samples were washed with icecold acetone and then tested using the BCA protein assay kit. CDDP for the preparation of LP-CDDP was purchased from JW Pharmaceutical Co. (Seoul, Republic of Korea). LP-CDDP was prepared by Katayama Chemical Industries, Co., Ltd., (Osaka, Japan) on a contract basis, and was formulated using dipalmitoylphosphatidylcholine, cholesterol, ganglioside, diacetyl phosphate and dipalmitoylphosphatidyl ethanolamine in the molar ratio of $35: 40: 15: 5: 5$. The lipid concentrations of LP-CDDP were measured as total cholesterol with $0.5 \%$ Triton X-100, using a Deternuber TC555 kit. The total lipid concentration was calculated by multiplying 2.5 by the cholesterol concentration. LP-CDDP was diluted to 10,000-fold with distilled water, and the concentration of platinum was measured using an automatic flameless atomic absorption spectrophotometer (FAAS) (Model AA-6700; Shimadzu, Kyoto, Japan) (26). Our previous studies demonstrated that, among the BNC-LP complexes prepared at $70^{\circ} \mathrm{C}$ and $\mathrm{pH} 3.0$, the highest yield of BNCs was obtained at a BNC:LP ratio of 1:20 [molar ratio of BNC (as particle):LP (as lipids) $=1: 1.6 \times 10^{4}$ or $1: 1.6 \times 10^{5}$, respectively] (21). To produce the BNC-LP-CDDP complex, LP-CDDP was incubated in Britton-Robinson buffer $\left(0.1 \mathrm{M} \mathrm{H}_{3} \mathrm{BO}_{3}, 0.1 \mathrm{M} \mathrm{CH}_{3} \mathrm{COOH}, 0.1 \mathrm{M} \mathrm{H}_{3} \mathrm{PO}_{4}\right.$ and $0.5 \mathrm{M}$ $\mathrm{NaOH}$; pH 3.0) at $70^{\circ} \mathrm{C}$ for 5 min, mixed with BNC, and then kept at $70^{\circ} \mathrm{C}$ for $60 \mathrm{~min}$ with gentle stirring. To exchange the buffer, the solutions were passed through a Sephadex G-25 (GE Healthcare, Buckinghamshire, UK) gel-filtration column with phosphate-buffered saline (PBS) at $\mathrm{pH}$ 7.4. The sizes and $\zeta$-potentials of the LP-CDDP and BNC-LP-CDDP were measured by dynamic light scattering (DLS) using a Zetasizer Nano ZS device (Malvern Instruments Ltd., Worcestershire, $\mathrm{UK})$. The polydispersity index (PDI) is a width parameter for the $\mathrm{Z}$-average as an intensity mean.

Cell culture. Human HCC Hep3B cells (ATCC no. HB-8064) were maintained in Dulbecco's modified Eagle's medium (Gibco-Invitrogen, Carlsbad, CA, USA). Human colorectal carcinoma HCT116 cells (ATCC no. CCL-247) were maintained in RPMI-1640 medium (Gibco-Invitrogen). Both media were supplemented with $10 \%$ fetal bovine serum (FBS; GibcoInvitrogen) and $1 \%$ penicillin/streptomycin (Gibco-Invitrogen) in a humidified atmosphere of $5 \% \mathrm{CO}_{2}$ at $37^{\circ} \mathrm{C}$.

Analysis of cell proliferation and cell cycle distribution. Hep3B and HCT116 cells in an exponential growth phase were harvested and plated in 96 -well plates $\left(5 \times 10^{3}\right.$ cells/well in $100 \mu \mathrm{l}$ of growth medium). Each experiment was performed in triplicate. Cells were exposed to CDDP, LP-CDDP, or BNC-LPCDDP at concentrations of $0,5,10$ and $15 \mu \mathrm{g} / \mathrm{ml}$ for $4 \mathrm{~h}$ and further incubated for $48 \mathrm{~h}$ without any drug. Cell proliferation assays were performed using the CCK- 8 assay kit (Dojindo Molecular Technologies, Gaithersburg, MD, USA) according to the manufacturer's instructions. For the cell cycle distribution assay, Hep3B cells were plated in a $60-\mathrm{mm}$ tissue culture dish at a density of $2 \times 10^{5}$ and incubated overnight. Cells were treated with $5 \mu \mathrm{g} / \mathrm{ml}$ of CDDP, LP-CDDP or BNC-LP-CDDP for $4 \mathrm{~h}$ and further incubated for $48 \mathrm{~h}$ without any drug. The cells were collected and suspended in $100 \%$ cold-ethanol for fixation. The fixed cells were stained with propidium iodide (PI), and the cell cycle distribution was then analyzed via flow cytometry (BD FACSCancto II).

Clonogenic survival assay. A clonogenic assay to determine the eternal proliferative potential of cells after treatment was performed by quantifying the colonies formed from single cells. Cells were plated in a 6 -well tissue culture plate at a density of 400, 800, 2,000 and 4,000 cells/well and were exposed to IR of $0,2,4$ and $8 \mathrm{~Gy}$, respectively. Cells were incubated in the 
presence of drugs such as CDDP, LP-CDDP, BNC-LP-CDDP, or BNC (equivalent to a CDDP concentration of $0.5 \mu \mathrm{g} / \mathrm{ml}$, $1.667 \mu \mathrm{M}$ ) for $4 \mathrm{~h}$, and were exposed IR in the absence of drugs. IR was delivered to the cell on plate using a 6-MV photon beam linear accelerator (CL/1800; Varian Medical Systems, Inc., Palo Alto, CA, USA). The cells were further incubated for $\sim 20$ days at $37^{\circ} \mathrm{C}$ for colony formation. Cells were stained with $0.5 \%$ crystal violet (Sigma-Aldrich, St. Louis, MO, USA) in $20 \%$ methanol. Stained colonies were washed with water, air-dried, and counted when they comprised $>50$ cells. The surviving fraction was calculated as mean colonies counted/ (cells plated x plating efficiency), where plating efficiency was defined as mean colonies counted/cells plated. All values were normalized to untreated cells. The mean number of colonies from triplicate tests, and their standard deviations were calculated. The sensitizer enhancement ratio (SER) was calculated as the radiation dose needed for radiation alone divided by the dose needed for various concentrations of drug plus radiation at a survival fraction of $0.5 \%$.

In vivo tumor growth delay. All animal experiments were performed following the protocol approved by the Institutional Animal Care and Use Committee of the Asan Institute for Life Science. The Hep3B cell-derived xenograft tumor model from male athymic nude mice (BALB/c-nude; 6-weeks old; Japan SLC Inc., Hamamatsu, Japan) was used to assess the in vivo therapeutic efficacy. To establish the xenograft model, a suspension of $3 \times 10^{6}$ cells was implanted subcutaneously (s.c) into the right hind leg of the mouse. The mice bearing xenograft tumors grown from 80 to $120 \mathrm{~mm}^{3}$ were pair-matched according to the tumor volume into experimental groups ( $\mathrm{n}=5$ in each group). Drugs were intravenously (i.v.) administered through the tail vein $2 \mathrm{~h}$ before IR treatment. A single dose of CDDP included $2 \mathrm{mg} / \mathrm{kg}$, and the doses of all other drugs were adjusted equivalent to the CDDP dose. The drug for administration was prepared at the concentration of $0.3 \mathrm{mg} / \mathrm{ml}$ and the volume given a mouse that was usually $\sim 150 \mu 1$ /mouse was proportional to the body weight. IR of 3 Gy was locally delivered to the tumor using a 6-MV photon beam linear accelerator, when mice were held in a mouse restrainer. The tumor volume and body weight were monitored during the entire experimental period. Tumor volume was calculated using the formula: Volume $=\left(\right.$ length $\mathrm{x}$ width $\left.{ }^{2}\right) \times 0.5$. The results are expressed as the mean \pm standard deviation.

Histological and immunohistochemical analysis. For immunohistochemical analysis of activated caspase-3, the Hep3B cell-derived xenograft mouse was i.v. injected with BNC,CDDP, LP-CDDP, or BNC-LP-CDDP at doses of $10 \mathrm{mg} / \mathrm{kg}$ depending on the amount of CDDP. After $2 \mathrm{~h}$, tumors were irradiated with 3 Gy using a 6-MV photon beam linear accelerator. Tumor tissues were collected after $24 \mathrm{~h}$ and fixed with $4 \%$ paraformaldehyde. The fixed tumor tissues were kept frozen in OCT compound and then cryosectionized using a cryotome. Immunohistochemical staining was performed with anti-cleaved-caspase-3 (\#9661; Cell Signaling Technology, Inc., Danvers, MA, USA) and was analyzed under a microscope (DP71; Olympus, Tokyo, Japan). The tumor tissues were counter-stained with hematoxylin. To assess nephrotoxicity, the mice were treated with CDDP, LP-CDDP, or BNC-LP-CDDP (equivalent to CDDP dose of
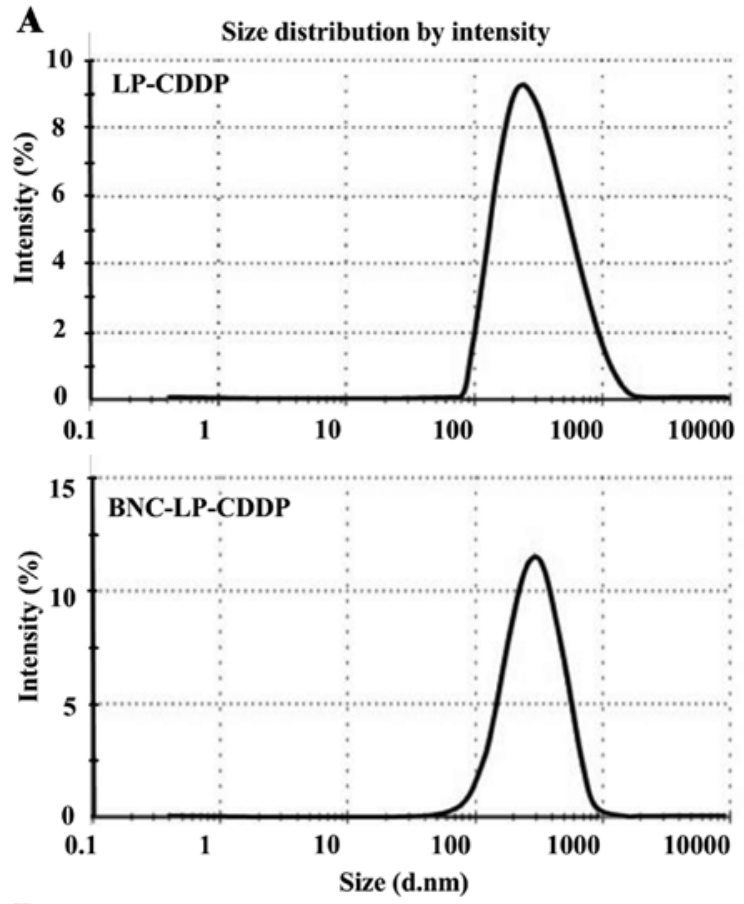

B

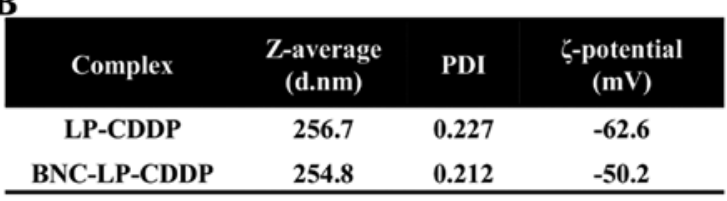

Figure 1. Characterization of LP-CDDP and BNC-LP-CDDP. (A) The size distribution of liposomal CDDP (LP-CDDP) and BNC-LP-CDDP measured with the DLS method. Scale bars, $100 \mathrm{~nm}$. (B) Physicochemical characterization of LP-CDDP and BNC-LP-CDDP.

$10 \mathrm{mg} / \mathrm{kg} ; \mathrm{n}=3$ ) and were sacrificed after 4 days. The kidney tissue was harvested, fixed in $4 \%$ paraformaldehyde, embedded in paraffin and sliced. The sections at a $5 \mu \mathrm{m}$-thickness were observed under a microscope (DP71; Olympus, Tokyo, Japan) after staining with hematoxylin and eosin.

\section{Results}

$H C C$-specific cytotoxicity of BNC-LP-CDDP. The size distribution of LP-CDDP and BNC-LP-CDDP was first analyzed using the DLS method, which indicated a value of $\sim 250 \mathrm{~nm}$ for both complexes (Fig. 1A). The material contents of LP-CDDP included $13.9 \mathrm{mg}$ of LP and $2.8 \mathrm{mg}$ of CDDP per ml, whereas that of BNC-LP-CDDP included $0.58 \mathrm{mg}$ of BNC, $7.6 \mathrm{mg}$ of $\mathrm{LP}$ and $1.5 \mathrm{mg}$ of CDDP per $\mathrm{ml}$ (Fig. 1B).

To ensure that BNC-LP-CDDP produces HCC-specific cytotoxicity, human $\mathrm{HCC}$ Hep3B cells and human colon cancer HCT116 cells were treated with BNC-LP-CDDP, and their viabilities were assessed. The viability of Hep3B treated with CDDP at 0, 5, 10 and $15 \mu \mathrm{g} / \mathrm{ml}$ was 100,61 and $43 \%$, respectively, whereas that of HCT116 in the same conditions was 100 , 57 and 43\%, respectively, thus, indicating that CDDP induced equivalent cytotoxicity in both cell lines (Fig. 2A and B). When the cells were treated with BNC-LP-CDDP, the Hep3B cells were more likely to die as compared to the HCT116 cells. The viability of Hep3B cells was reduced to $34 \%$ following BNC-LP-CDDP treatment at $15 \mu \mathrm{g} / \mathrm{ml}$, in contrast to the 

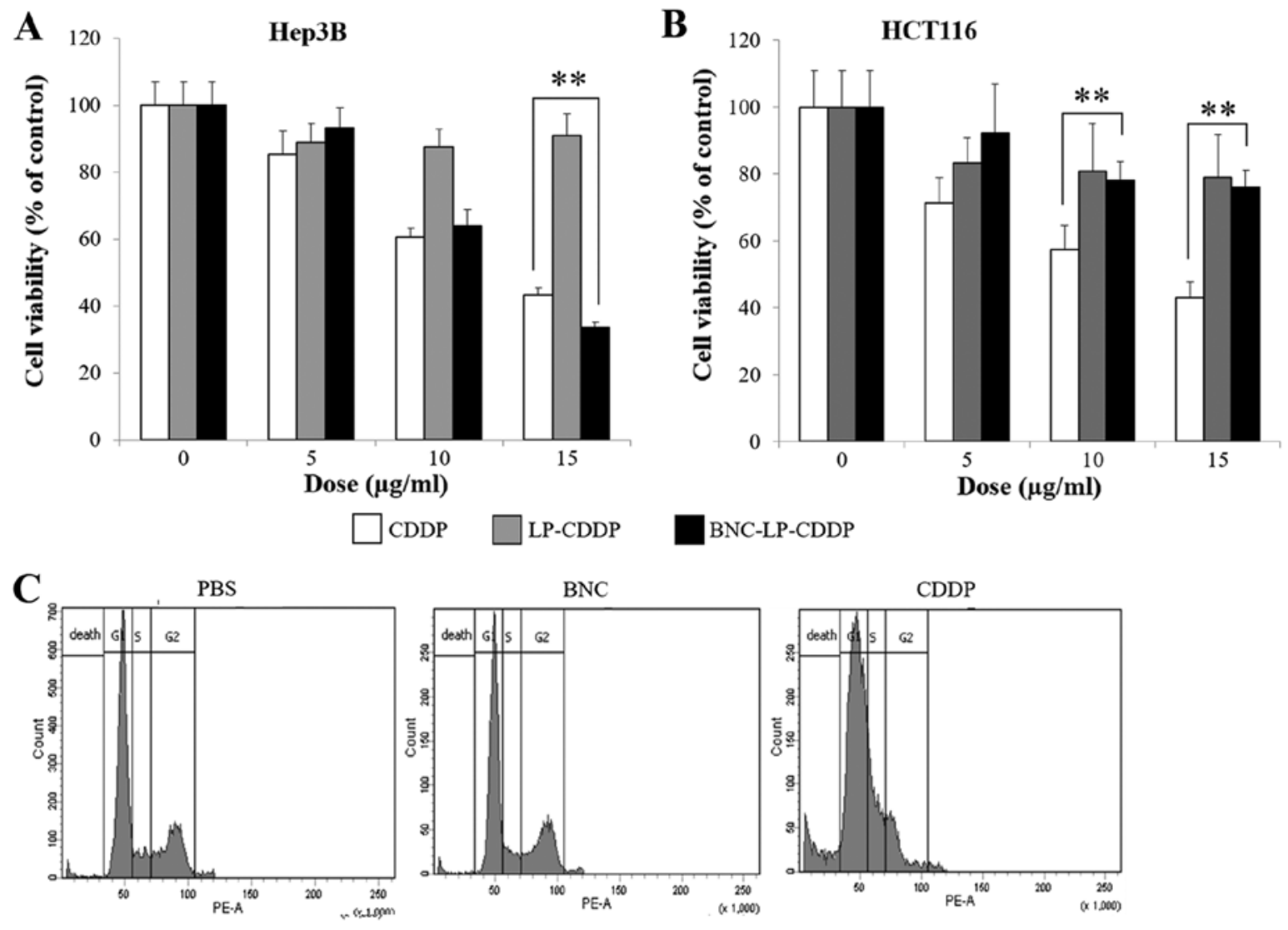

CDDP $\square$ LP-CDDP

BNC-LP-CDDP
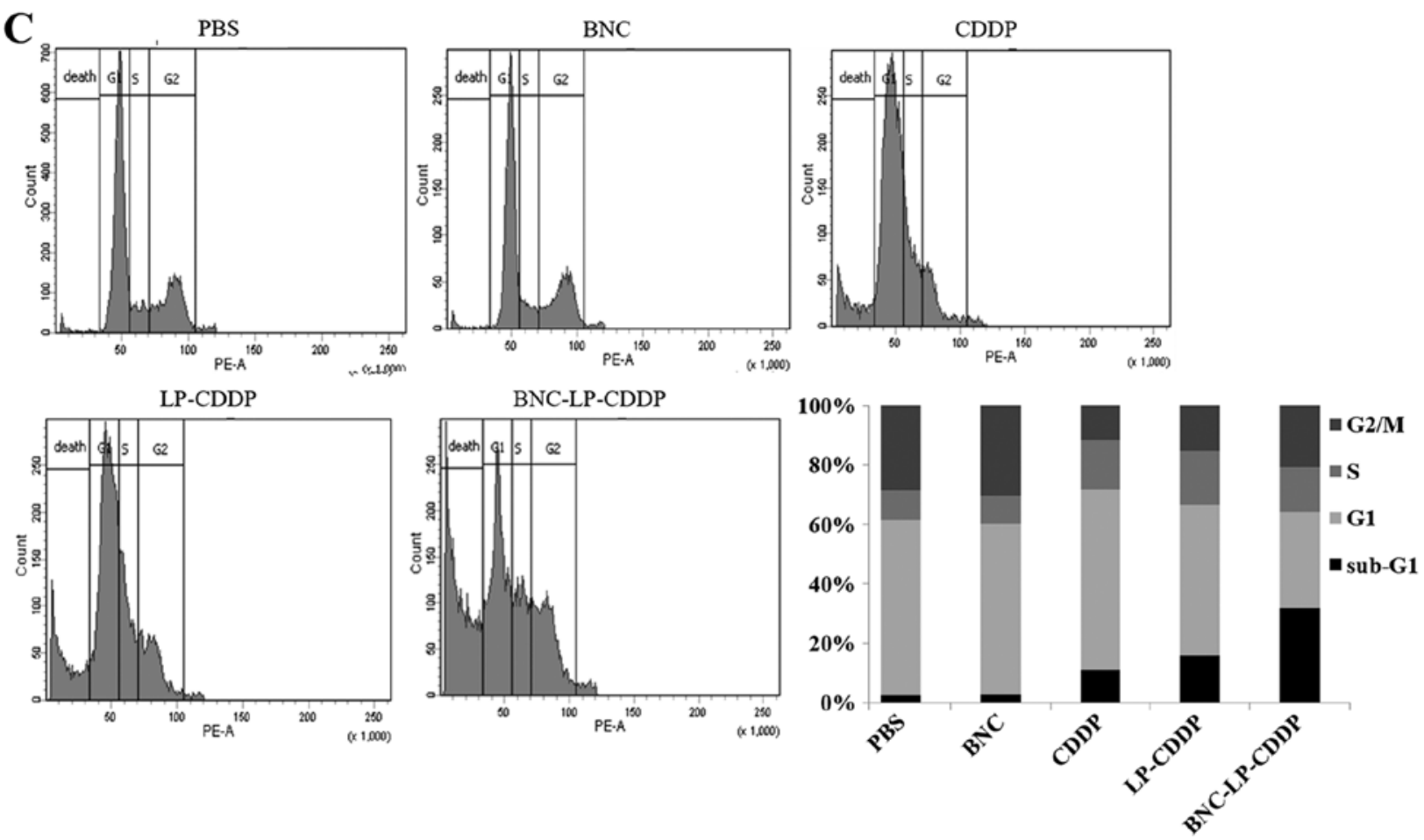

Figure 2. HCC-specific cytotoxicity of BNC-LP-CDDP. (A and B) Cell viability assay. Hep3B (HCC) (A) and HCT116 (colon cancer) (B) cells were treated with cis-diamminedichloroplatinum (II) (CDDP), liposomal CDDP (LP-CDDP), or BNC-LP-CDDP at different concentrations $(0,10 \mathrm{or} 15 \mu \mathrm{g} / \mathrm{ml})$ for $4 \mathrm{~h}$ After incubation for $48 \mathrm{~h}$ without any drug, cellular viability was measured using the Cell Counting kit- 8 . Data are presented as mean \pm standard deviation. ${ }^{* *} \mathrm{P}<0.001$. (C) Cell cycle distribution. Hep3B cells were treated with PBS, BNC, CDDP, LP-CDDP, or BNC-LP-CDDP at $5 \mu \mathrm{g} / \mathrm{ml}$ for $4 \mathrm{~h}$. After incubation for $48 \mathrm{~h}$ without any drug, cells were subjected to FACS analysis and were stained with propidium iodide (PI), and the DNA contents were measured using flow cytometry.

viability of $76 \%$ in HCT116 cells following BNC-LP-CDDP treatment. These results suggest that BNC-LP-CDDP specifically targets and kills HCC cells.

To examine the effect of BNC-LP-CDDP on cell cycle distribution and the sub-G1 population of Hep3B cells, the cells were exposed to a lower concentration $(5 \mu \mathrm{g} / \mathrm{ml})$ of drugs and were subjected to analysis of the DNA contents using flow cytometry. We found that BNC-LP-CDDP treatment markedly increased the sub-G1 population of the cells to $31.3 \%$, whereas CDDP and LP-CDDP treatment resulted in a lower sub-G1 population. These results indicate that BNC-LP-CDDP strongly induces cell death in human HCC cells.
Radiosensitization of HCC cells by BNC-LP-CDDP. To assess the effect of BNC-LP-CDDP on the in vitro chemoradiotherapeutic efficacy, Hep3B cells were treated with BNC, CDDP, LP-CDDP, or BNC-LP-CDDP at a lower concentration (such as $0.5 \mu \mathrm{g} / \mathrm{ml}$ ) and were exposed to IR at $0,2,4$ or $8 \mathrm{~Gy}$. In the clonogenic assay, the survival fractions of the cells without drug treatment were estimated as follows: 1, 0.56, 0.46 and 0.06 at radiation doses of $0,2,4$ and $8 \mathrm{~Gy}$, respectively (Fig. 3). The survival fraction of cells was most effectively reduced when the cells were pre-treated with BNC-LP-CDDP, which led to survival fractions of 1, 0.15, 0.09 and 0.02 at $0,2,4$, and $8 \mathrm{~Gy}$, respectively. The sensitizer enhancement ratio (SER) of BNC-LP-CDDP, LP-CDDP and CDDP were 5.83, 

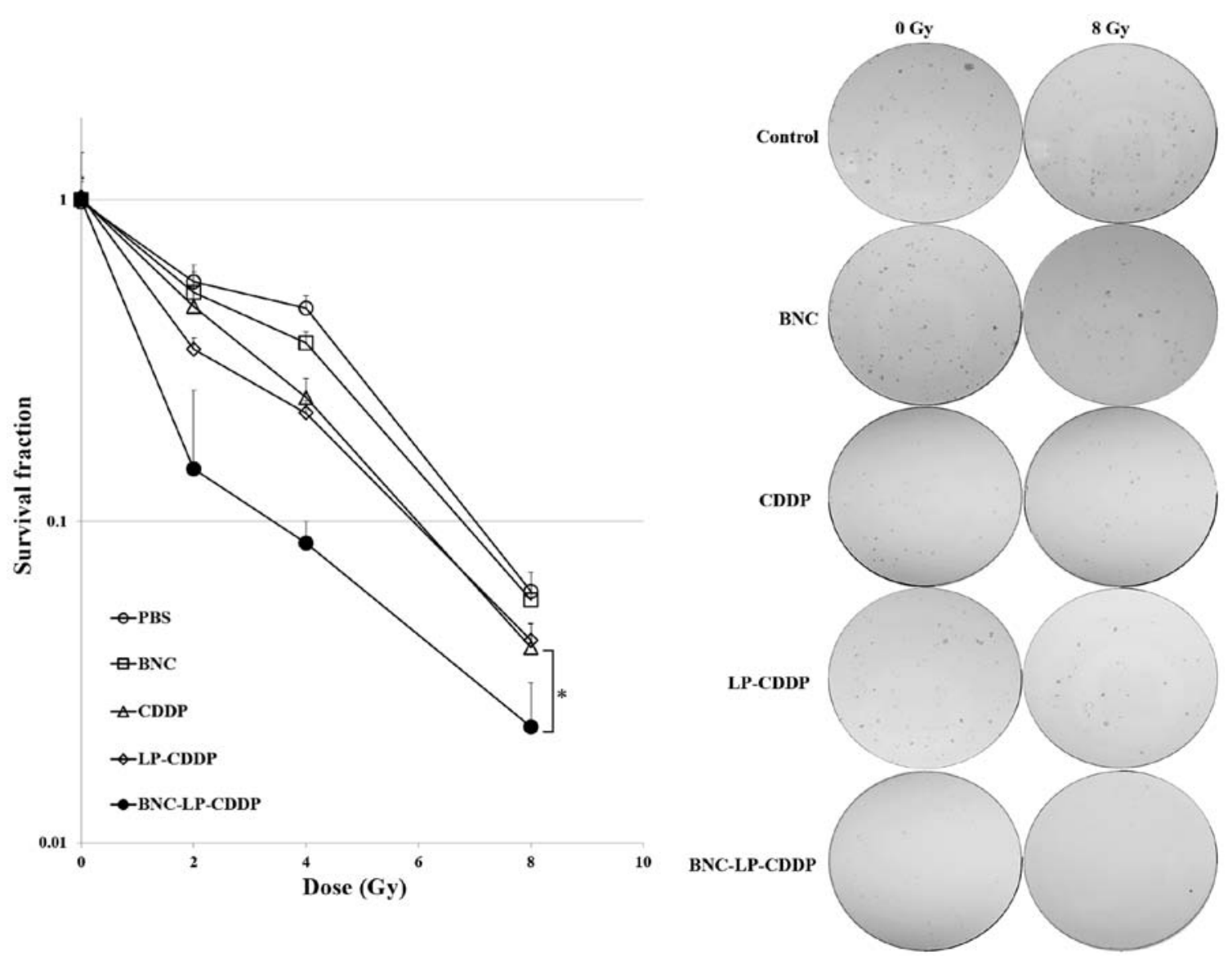

Figure 3. Radiosensitization effect of BNC-LP-CDDP. Hep3B cells were treated with PBS, BNC, CDDP, liposomal CDDP (LP-CDDP), or BNC-LP-CDDP. The cells were irradiated after $4 \mathrm{~h}$, and were then incubated until colonies comprising $>50$ cells were obtained. The survival fraction for each sample was normalized to the survival fraction of untreated controls. Data are presented as the mean \pm standard deviation. ${ }^{*} \mathrm{P}<0.01$.

2.12 and 1.41 , respectively, compared to that with IR alone. These results suggest that BNC-LP-CDDP potentially exerted radiosensitization effects in $\mathrm{HCC}$ cells.

Enhancement of radiotherapeutic efficacy by BNC-LP-CDDP in vivo. As BNC-LP-CDDP showed in vitro radiosensitization effects in HCC cells, we then examined the in vivo effect of BNC-LP-CDDP on the chemoradiotherapeutic efficacy in mice bearing Hep3B xenograft tumors, compared with that of CDDP or LP-CDDP. The mice were i.v. injected with CDDP, LP-CDDP, or BNC-LP-CDDP (single dose, $2 \mathrm{mg} / \mathrm{kg}$; equivalent to the amount of CDDP) $2 \mathrm{~h}$ before IR at $3 \mathrm{~Gy}$. Tumor growth in the CDDP-, LP-CDDP- or BNC-LP-CDDP-treated mice without IR irradiation was delayed, as compared to that in the control group (Fig. 4A). On day 32, the treated/control tumor size $(\mathrm{T} / \mathrm{C})$ percentage values of the control, CDDP-, LP-CDDP- and BNC-LP-CDDP-treated groups were 100, 66, 73.6 and $56 \%$, indicating that BNC-LP-CDDP exerted better in vivo therapeutic efficacy than CDDP or LP-CDDP, even without combination treatment with IR (Table I). When the treatment was combined with IR, the T/C percentage values of IR alone, CDDP, LP-CDDP and BNC-LP-CDDP treatment on day 32 were estimated as $34.4,24.6,26.4$ or $4.3 \%$, respectively (Table I). These results suggest that BNC-LP-CDDP displays potent radiosensitization effects in vivo and significantly enhances the chemoradiotherapeutic efficacy. An equal amount of BNC and BNC-LP-CDDP was tested under similar
Table I. The treated/control tumor size (T/C) percentage values.

\begin{tabular}{lc} 
Treatment & TGI \% (Day 32) \\
\hline PBS & 100 \\
CDDP & 66.0 \\
LP-CDDP & 73.6 \\
BNC-LP-CDDP & 56.0 \\
IR & 34.4 \\
CDDP + IR & 24.6 \\
LP-CDDP + IR & 26.4 \\
BNC-LP-CDDP + IR & 4.3 \\
\hline
\end{tabular}

TGI, tumor growth inhibition; IR, irradiation.

experimental conditions to confirm the effect on radiotherapy. BNC did not affect tumor growth or radiosensitivity (Fig. 4C). Considerable changes in body weight were not observed in this experiment, indicating that none of the mice suffered from severe toxicity (Fig. 4B and D). Thus, these results clearly demonstrate that BNC-LP-CDDP effectively enhances the chemoradiotherapeutic efficacy in vivo without severe toxicity.

Increased apoptosis induction by BNC-LP-CDDP in vivo. To determine whether BNC-LP-CDDP induces more apoptosis 

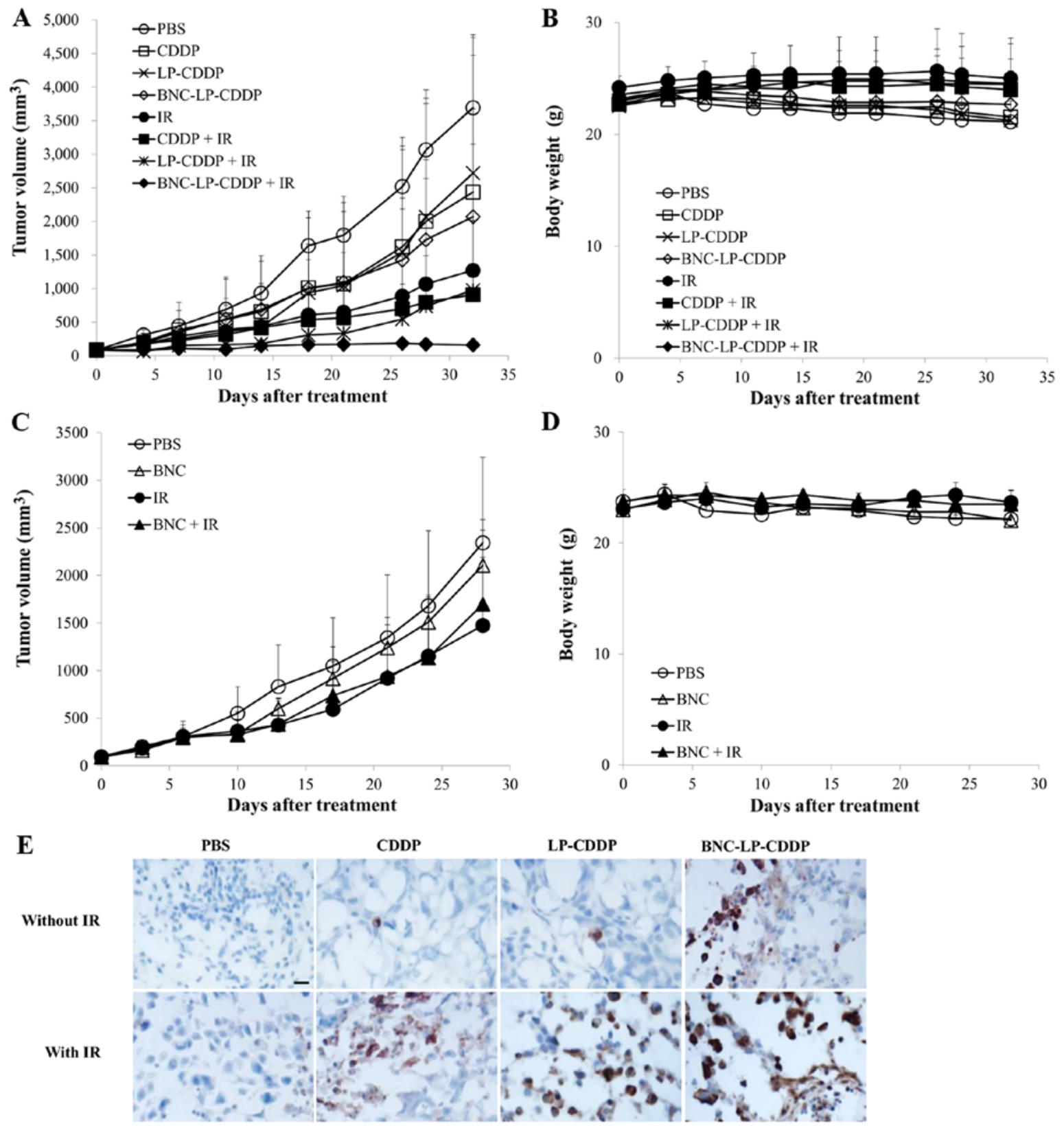

Figure 4. In vivo radiosensitization effect and apoptosis induction by BNC-LP-CDDP. (A-D) Tumor growth delay curves (A, C) and changes in body weight (B and D). Mice bearing the Hep3B tumor were intravenously administered with CDDP, liposomal CDDP (LP-CDDP), BNC-LP-CDDP (A), or BNC (C) at a single $2 \mathrm{mg} / \mathrm{kg}$ dose based on the amount of CDDP. After $2 \mathrm{~h}$, tumors were irradiated with IR at $3 \mathrm{~Gy}$, and the tumor volumes and body weights were monitored during the experimental course. Data are presented as the mean \pm standard deviation. (E) Mice bearing the Hep3B tumor were treated intravenously with CDDP, liposomal CDDP (LP-CDDP), and BNC-LP-CDDP at a single $10 \mathrm{mg} / \mathrm{kg}$ dose based on the amount of CDDP. After 1 day, the tumors were harvested and cryo-sectioned. DAB images of immunohistochemically stained activated caspase- 3 were obtained under a microscope. Scale bar, $20 \mu \mathrm{m}$.

than other drug, the levels of activated caspase-3 in tumor tissue were estimated via immunohistochemistry. As shown in Fig. 4E, BNC-LP-CDDP without IR induced greater apoptosis in tumor tissue as compared to CDDP alone or LP-CDDP alone. Notably, BNC-LP-CDDP with IR significantly increased the cleavage of caspase- 3 as compared to other drugs. These results suggest that BNC-LP-CDDP effectively induced apoptosis in tumor tissue, which led to greater enhancement of the in vivo therapeutic efficacy.

Withdrawal of nephrotoxicity by BNC-LP-CDDP. The sideeffects of CDDP, including nephrotoxicity, are well known, and hence, we investigated whether BNC-LP-CDDP led to the withdrawal of CDDP-induced nephrotoxicity through a histopathological examination in the mice. Mice were injected with a single $10-\mathrm{mg} / \mathrm{kg}$ dose of CDDP, LP-CDDP, or BNC-LP-CDDP. After 4 days, the kidney tissue was collected and stained with hematoxylin and eosin. Acute cortical tubular degeneration and regeneration were observed in the kidney in CDDP-treated animals (Fig. 5B). In contrast, the kidneys of mice treated with BNC-LP-CDDP did not show any observable toxic damage (Fig. 5D), similar to those treated with LP-CDDP (Fig. 5C). These results indicate that BNC-LP-CDDP did not exhibit the nephrotoxic properties of CDDP, potentially due to the encapsulation of CDDP into the LP. 

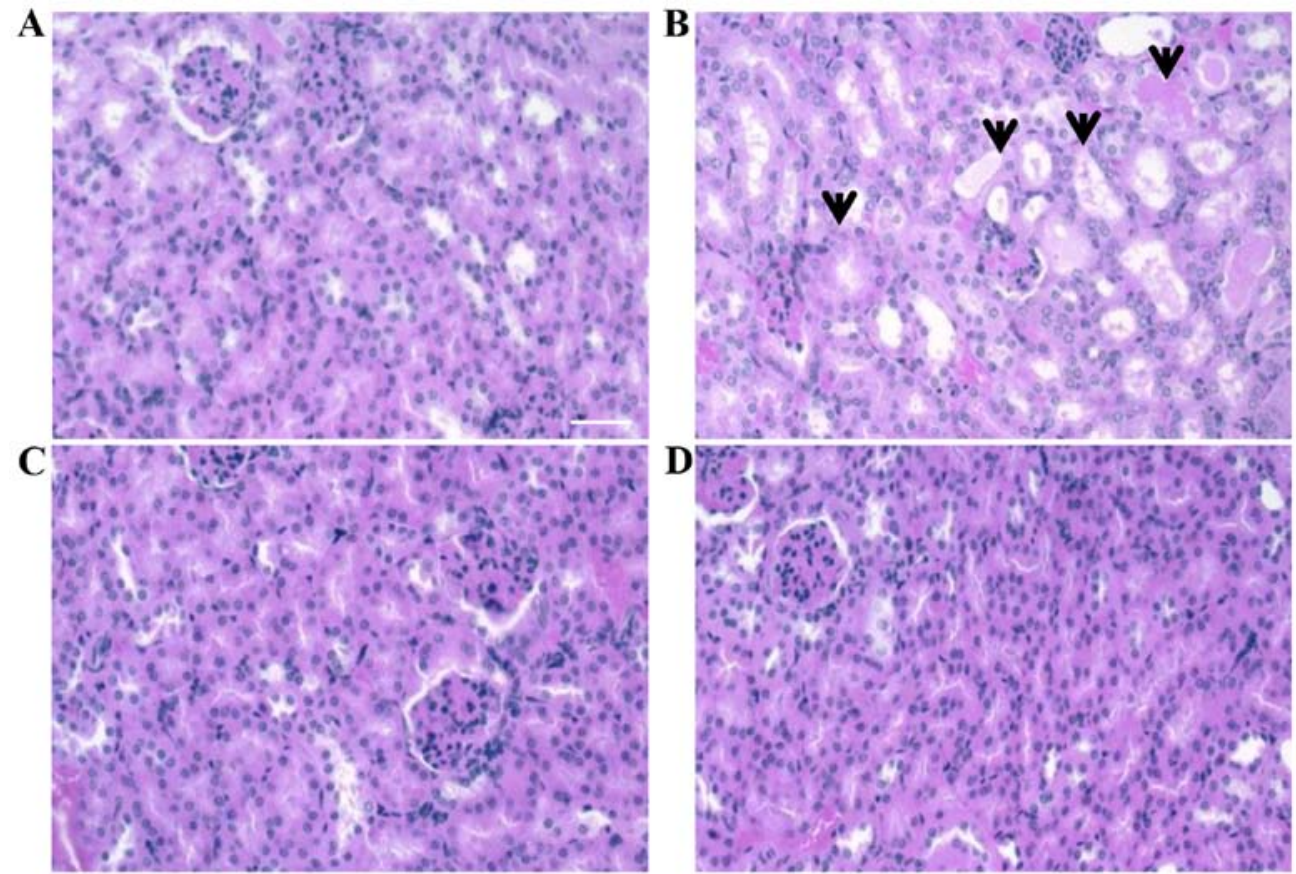

Figure 5. Withdrawal of nephrotoxicity by BNC-LP-CDDP. Mice bearing the Hep3B tumor were treated intravenously with PBS (A), CDDP (B), liposomal CDDP (LP-CDDP) (C), or BNC-LP-CDDP (D) at a single $10 \mathrm{mg} / \mathrm{kg}$ dose based on the amount of CDDP. After 4 days, kidneys were harvested, embedded in paraffin and sectioned. The hematoxylin and eosin-stained tissues were analyzed under a microscope. Scale bar, $50 \mu \mathrm{m}$.

\section{Discussion}

$\mathrm{HCC}$ is the most common cause of cancer-related death, particularly in Asia. Moreover, HCC is known to be resistant to the currently available chemotherapeutic agents and radiotherapy $(1,2,27)$. In the present study, we introduce BNC-LP-CDDP as a candidate for overcoming the limitations in the current treatment of human HCC. The use of LPs as carriers of drugs or genes has been attempted in anticancer therapy $(22,23)$. For instance, Doxil, wherein doxorubicin is incorporated in an LP, was the first nano anticancer drug to be approved by the FDA (28). Although several nanodrugs have been approved since then, no agent that specifically targets $\mathrm{HCC}$ has been identified. We previously developed a BNC and reported on its ability to target liver as well as HCC, as LPs serve as a versatile form of drug delivery system for carrying genes or drugs (29-31). Furthermore, hepatitis B virus (HBV) infects liver cells only in humans and chimpanzees, and not in other animals. The hepatophilicity is determined by the pre-S regions (pre-S1, 2) at the N-terminal half of the HBV surface antigen L-protein. Furthermore, pre-S1 is indispensable for the specific binding of HBV to hepatocytes, whereas pre-S2 in necessary for both the poly-albumin-mediated cell attachment of HBV and cell permeability of HBV (30). In the present study, we described the properties of BNC-LP-CDDP along with pre-S1 in L-protein as a promising treatment strategy and the potency of BNC-LP-CDDP as radiosensitizer for $\mathrm{HCC}$ through in vitro and in vivo experiments in human $\mathrm{HCC}$ models. In results, BNC-LP-CDDP targeted specifically to human HCC Hep3B cells both in vitro (Figs. 2 and 3) and in vivo (Fig. 4), and induced potent apoptosis that led to a marked enhancement of therapeutic efficacy, of which results were consistently observed in other hepatoma cell lines including Huh7 or HepG2 as reported previously by us $(29,31)$. According to the reports, we concluded through repeated experiment that $\mathrm{BNC}$ targeted $\mathrm{HCC}$ better than normal liver tissue (29). Most importantly, BNC-LP-CDDP displayed great radiosensitization effects via the active-targeted delivering of CDDP, thus simultaneously eliminating the nephrotoxicity induced by CDDP (Fig. 5). Given that CDDP is both a competent radiosensitizer and toxic to the kidney, BNC-LP-CDDP could serve as a promising therapeutic option and present a new conceptual modality, such as chemoradiotherapy with active targeting nanomedicine, for human HCC treatment.

With regard to diverse utilizations of BNC for targeting other sites, BNC has been modified as zz-BNC harboring the protein-A Z domain (Staphylococcus aureus), LL-BNC harboring the protein-L B1 domain (Finegoldia magna), or LG-BNC harboring the protein-L B1 domain and the proteinG C2 domain (Streptococcus species) by replacing the domain indispensable for the human hepatotrophic property of BNC. Thus, BNC can be modified to recognize specific target cells in an antibody-dependent manner by displaying IgGs such as human $\operatorname{IgG} 3$, human $\operatorname{IgM}$, mouse $\operatorname{IgG} 1$ and rat $\operatorname{IgG}$ on the modified BNC $(29,32,33)$. Thus, BNC may represent a promising active targeting-based drug delivery system for not only HCC, but also other types of human cancers.

Radiotherapy is a common strategy that is used for the treatment of diverse cancer types. Above all, radiotherapy for liver tumor has been performed historically and currently through diverse strategy including three-dimensional conformal radiation therapy (3D-CRT), stereotactic body radiation therapy (SBRT) and transarterial radioembolization (TARE) (34). Concurrent chemoradiotherapy can also enhance the cure rate of patients resistant to other therapies. Doxorubicin, gemcitabine, docetaxel, paclitaxel and CDDP are widely known drugs for 
chemoradiotherapy. Among these drugs, CDDP is known to be the most potent radiosensitizer in a wide array of cancer types, although it cannot be currently used as chemoradiotherapy for HCC due to the accompanied side-effects. We have confirmed that BNC-LP-CDDP overcame the original nephrotoxicity due to CDDP via histopathological analysis. A preclinical toxicology study regarding the successful clinical application of BNC-LP-CDDP is currently ongoing.

\section{Acknowledgements}

The present study was supported by grants from the Korean Health Technology R\&D Project through the Korea Health Industry Development Institute (KHIDI), funded by the Ministry for Health \& Welfare, Republic of Korea (HI06C0868 and HI15C0972); the National R\&D Program for Cancer Control, Ministry of Health \& Welfare, Republic of Korea (15201101); and the Basic Science Research Program through the National Research Foundation of Korea (NRF) funded by the Ministry of Education (NRF-2017R1D1A1B03034359 and NRF-2017R1D1A1B03035167).

\section{References}

1. Llovet JM, Burroughs A and Bruix J: Hepatocellular carcinoma. Lancet 362: 1907-1917, 2003

2. Jemal A, Bray F, Center MM, Ferlay J, Ward E and Forman D: Global cancer statistics. CA Cancer J Clin 61: 69-90, 2011.

3. Yau T, Chan P, Ng KK, Chok SH, Cheung TT, Fan ST and Poon RT: Phase 2 open-label study of single-agent sorafenib in treating advanced hepatocellular carcinoma in a hepatitis B-endemic Asian population: Presence of lung metastasis predicts poor response. Cancer 115: 428-436, 2009.

4. Mendez Romero A and Høyer M: Radiation therapy for liver metastases. Curr Opin Support Palliat Care 6: 97-102, 2012.

5. Gallicchio R, Nardelli A, Mainenti P, Nappi A, Capacchione D, Simeon V, Sirignano C, Abbruzzi F, Barbato F, Landriscina M, et al: Therapeutic strategies in HCC: Radiation modalities. BioMed Res Int 2016: 1295329, 2016.

6. Harris AL: Hypoxia: a key regulatory factor in tumour growth. Nat Rev Cancer 2: 38-47, 2002.

7. Barker HE, Paget JT, Khan AA and Harrington KJ: The tumour microenvironment after radiotherapy: Mechanisms of resistance and recurrence. Nat Rev Cancer 15: 409-425, 2015.

8. Jothy Basu KS, Bahl A, Subramani V, Sharma DN, Rath GK and Julka PK: Normal tissue complication probability of fibrosis in radiotherapy of breast cancer: Accelerated partial breast irradiation vs conventional external-beam radiotherapy. J Cancer Res Ther 4: 126-130, 2008.

9. Jeong SY1, Park SJ, Yoon SM, Jung J, Woo HN, Yi SL, Song SY, Park HJ, Kim C, Lee JS, et al: Systemic delivery and preclinical evaluation of $\mathrm{Au}$ nanoparticle containing beta-lapachone for radiosensitization. J Control Release 139: 239-245, 2009.

10. Jung J, Park SJ, Chung HK, Kang HW, Lee SW, Seo MH, Park HJ, Song SY, Jeong SY and Choi EK: Polymeric nanoparticles containing taxanes enhance chemoradiotherapeutic efficacy in non-small cell lung cancer. Int J Radiat Oncol Biol Phys 84: e77-e83, 2012.

11. Woo HN, Chung HK, Ju EJ, Jung J, Kang HW, Lee SW, Seo MH, Lee JS, Lee JS, Park HJ, et al: Preclinical evaluation of injectable sirolimus formulated with polymeric nanoparticle for cancer therapy. Int J Nanomed 7: 2197-2208, 2012.

12. Yoshikawa $\mathrm{M}$, Ono $\mathrm{N}$, Yodono $\mathrm{H}$, Ichida $\mathrm{T}$ and Nakamura $\mathrm{H}$ Phase II study of hepatic arterial infusion of a fine-powder formulation of cisplatin for advanced hepatocellular carcinoma. Hepatol Res 38: 474-483, 2008.

13. Pottier A, Borghi E and Levy L: New use of metals as nanosized radioenhancers. Anticancer Res 34: 443-453, 2014.

14. Dasari S and Tchounwou PB: Cisplatin in cancer therapy: Molecular mechanisms of action. Eur J Pharmacol 740: 364-378, 2014.
15. Yao X, Panichpisal K, Kurtzman N and Nugent K: Cisplatin nephrotoxicity: A review. Am J Med Sci 334: 115-124, 2007.

16. Pabla N and Dong Z: Cisplatin nephrotoxicity: Mechanisms and renoprotective strategies. Kidney Int 73: 994-1007, 2008.

17. Zhang X, Yang H, Gu K, Chen J, Rui M and Jiang GL: In vitro and in vivo study of a nanoliposomal cisplatin as a radiosensitizer. Int J Nanomed 6: 437-444, 2011

18. Guo S, Wang Y, Miao L, Xu Z, Lin CM, Zhang Y and Huang L: Lipid-coated Cisplatin nanoparticles induce neighboring effect and exhibit enhanced anticancer efficacy. ACS Nano 7: 9896-9904, 2013.

19. Yamada T, Iwabuki H, Kanno T, Tanaka H, Kawai T, Fukuda H, Kondo A, Seno M, Tanizawa K and Kuroda S: Physicochemical and immunological characterization of hepatitis B virus envelope particles exclusively consisting of the entire L (pre-S1 + pre-S2 + S) protein. Vaccine 19: 3154-3163, 2001.

20. Nagaoka T, Fukuda T, Yoshida S, Nishimura H, Yu D, Kuroda S, Tanizawa K, Kondo A, Ueda M, Yamada H, et al: Characterization of bio-nanocapsule as a transfer vector targeting human hepatocyte carcinoma by disulfide linkage modification. J Control Release 118: 348-356, 2007.

21. Liu Q, Jung J, Somiya M, Iijima M, Yoshimoto N, Niimi T, Maturana AD, Shin SH, Jeong SY, Choi EK, et al: Virosomes of hepatitis B virus envelope L proteins containing doxorubicin: Synergistic enhancement of human liver-specific antitumor growth activity by radiotherapy. Int J Nanomed 10: 4159-4172, 2015.

22. Allen TM and Cullis PR: Liposomal drug delivery systems: From concept to clinical applications. Adv Drug Deliv Rev 65: 36-48, 2013.

23. Drummond DC, Meyer O, Hong K, Kirpotin DB and Papahadjopoulos D: Optimizing liposomes for delivery of chemotherapeutic agents to solid tumors. Pharmacol Rev 51: 691-743, 1999.

24. Deshpande PP, Biswas S and Torchilin VP: Current trends in the use of liposomes for tumor targeting. Nanomedicine (Lond) 8: 1509-1528, 2013.

25. Jung J, Iijima M, Yoshimoto N, Sasaki M, Niimi T, Tatematsu K, Jeong SY, Choi EK, Tanizawa K and Kuroda S: Efficient and rapid purification of drug- and gene-carrying bio-nanocapsules, hepatitis B virus surface antigen L particles, from Saccharomyces cerevisiae. Protein Expr Purif 78: 149-155, 2011.

26. Hirai M, Minematsu H, Hiramatsu Y, Kitagawa H, Otani T, Iwashita S, Kudoh T, Chen L, Li Y, Okada M, et al: Novel and simple loading procedure of cisplatin into liposomes and targeting tumor endothelial cells. Int J Pharm 391: 274-283, 2010.

27. Maluccio $M$ and Covey A: Recent progress in understanding, diagnosing, and treating hepatocellular carcinoma. CA Cancer J Clin 62: 394-399, 2012.

28. Dawidczyk CM, Kim C, Park JH, Russell LM, Lee KH, Pomper MG and Searson PC: State-of-the-art in design rules for drug delivery platforms: Lessons learned from FDA-approved nanomedicines. J Control Release 187: 133-144, 2014.

29. Jung J, Matsuzaki T, Tatematsu K, Okajima T, Tanizawa K and Kuroda S: Bio-nanocapsule conjugated with liposomes for in vivo pinpoint delivery of various materials. J Control Release 126: 255-264, 2008

30. Kasuya T, Yamada T, Uyeda A, Matsuzaki T, Okajima T, Tatematsu K, Tanizawa K and Kuroda S: In vivo protein delivery to human liver-derived cells using hepatitis B virus envelope pre-S region. J Biosci Bioeng 106: 99-102, 2008.

31. Kasuya T, Jung J, Kinoshita R, Goh Y, Matsuzaki T, Iijima M, Yoshimoto N, Tanizawa K and Kuroda S: Chapter 8 Bio-nanocapsule-liposome conjugates for in vivo pinpoint drug and gene delivery. Methods Enzymol 464: 147-166, 2009.

32. Tatematsu K, Iijima M, Yoshimoto N, Nakai T, Okajima T and Kuroda S: Bio-nanocapsules displaying various immunoglobulins as an active targeting-based drug delivery system. Acta Biomater 35: 238-247, 2016

33. Tsutsui Y, Tomizawa K, Nagita M, Michiue H, Nishiki T, Ohmori I, Seno M, Matsui H, et al: Development of bionanocapsules targeting brain tumors. J Control Release 122: 159-164, 2007.

34. Tanguturi SK, Wo JY, Zhu AX, Dawson LA and Hong TS: Radiation therapy for liver tumors: Ready for inclusion in guidelines? Oncologist 19: 868-879, 2014. 